\title{
CERTAIN SELF-RECIPROCAL FUNCTIONS
}

BRIJ MOHAN

In 1932 and 1933 I [5, 6] gave some rules connecting different classes of self-reciprocal functions. The object of this note is to derive some new self-reciprocal functions with the help of those rules.

I will say that a function is $R_{\nu}$ if it is self-reciprocal for $J_{\nu}$ transforms, where $\nu>-1$.

I will make use of the following results given in the papers referred to:

If $f(x)$ is $R_{\mu}$, the functions $g(x)$ given by the following integral formulas are all $R_{\nu}$ :

$$
\begin{aligned}
& g(x)=x^{(\nu-\mu+1) / 2} \int_{0}^{\infty} y^{(\nu-\mu+1) / 2} J_{(\mu+\nu) / 2}(x y) f(y) d y, \\
& g(x)=x^{(\mu-\nu+1) / 2} \int_{0}^{\infty} y^{(\mu-\nu+1) / 2} J_{(\mu+\nu) / 2}(x y) f(y) d y, \\
& g(x)=\int_{0}^{\infty} \frac{y^{\mu+1 / 2} f(x y)}{\left(1+y^{2}\right)^{1+\mu / 2+\nu / 2}} d y, \\
& g(x)=\int_{1}^{\infty} \frac{y^{1 / 2-\mu} f(x y)}{\left(y^{2}-1\right)^{1-\mu / 2+\nu / 2}} d y, \\
& g(x)=\int_{0}^{1} \frac{y^{1 / 2+\mu} f(x y)}{\left(1-y^{2}\right)^{1+\mu / 2-\nu / 2}} d y .
\end{aligned}
$$

If, in (ii) we take the familiar $R_{\mu}$ function

$$
x^{\mu+1 / 2} e^{-x^{2} / 2}
$$

for $f(x)$, we get

$$
\begin{aligned}
g(x) & =x^{(\mu-\nu+1) / 2} \int_{0}^{\infty} y^{(\mu-\nu+1) / 2} J_{(\mu+\nu) / 2}(x y) \cdot y^{\mu+1 / 2} e^{-y^{2} / 2} d y \\
& =x^{(\mu-\nu+1) / 2} \int_{0}^{\infty} y^{3 \mu / 2-\nu / 2+1} e^{-y^{2} / 2} J_{\mu / 2+\nu / 2}(x y) d y .
\end{aligned}
$$

Evaluating this integral by Hankel's formula [7], we get

$$
\begin{aligned}
g(x)=x^{(\mu-\nu+1) / 2} & \frac{\Gamma(\mu+1)\left(x / 2^{1 / 2}\right)^{\mu / 2+\nu / 2}}{2^{-3 \mu / 4+\nu / 4} \Gamma(1+\mu / 2+\nu / 2)} e^{-x^{2} / 2} \\
& \cdot{ }_{1} F_{1}\left(\nu / 2-\mu / 2 ; 1+\mu / 2+\nu / 2 ; x^{2} / 2\right), \quad \mu>-1 .
\end{aligned}
$$


This shows that the function $x^{\mu+1 / 2} e^{-x^{2} / 2}{ }_{1} F_{1}(\nu / 2-\mu / 2 ; 1+\mu / 2+\nu / 2$; $\left.x^{2} / 2\right),(\mu>-1)$, which is the same as.

$$
x^{\nu+2 n+1 / 2} e^{-x^{2} / 2} F_{1}\left(-n ; n+\nu+1 ; x^{2} / 2\right), \quad \nu+2 n>-1,
$$

is $R_{\nu}$.

Particular cases. $(\alpha)$ Since, when $n$ is a positive integer,

$$
L_{n}^{(\alpha)}(x)=\frac{\Gamma(1+\alpha+n)}{n ! \Gamma(\alpha+1)}{ }_{1} F_{1}(-n ; \alpha+1 ; x),
$$

where $L_{n}^{(\alpha)}(x)$ denotes the generalized Laguerre polynomial of order $n$, it follows that the function $x^{\nu+2 n+1 / 2} e^{-x^{2} / 2} L_{n}^{(n+\nu)}\left(x^{2} / 2\right)$, $(\nu+2 n>-1)$, is $R_{\nu}$ for positive integral values of $n$.

This result was given by Howell [4].

$(\beta)$ Since, when $n$ is a positive integer,

$$
T_{\mu}^{n}(x)=\frac{(-1)^{n}}{n ! \Gamma(1+\mu)}{ }_{1} F_{1}(-n ; 1+\mu ; x),
$$

where $T_{\mu}^{n}(x)$ is Sonine's polynomial of order $n$, it follows that the function

$$
x^{\nu+2 n+1 / 2} e^{-x^{2} / 2} T_{n+\nu}^{n}\left(x^{2} / 2\right), \quad \quad \nu+2 n>-1,
$$

is $R_{\nu}$ for positive integral values of $n$.

$(\gamma)$ If we put $n=-\nu-1 / 2$, our function becomes $x^{-\nu-1 / 2} e^{-x / 2}$ ${ }_{1} F_{1}\left(\nu+1 / 2 ; 1 / 2 ; x^{2} / 2\right)$.

As a particular case, when $\nu$ is an integer, this becomes a constant multiple of

$$
x^{-\nu-1 / 2} e^{-x^{2} / 4} D_{-2 \nu-1}(x) .
$$

( $\delta$ ) When $n=1 / 2-\nu$, the function is $x^{-\nu+3 / 2} e^{-x^{2} / 2}{ }_{1} F_{1}(\nu-1 / 2 ; 3 / 2$; $\left.x^{2} / 2\right),(\nu<2)$.

If $\nu$ is an integer, this is a constant multiple of

$$
x^{-p+1 / 2} e^{-x^{2} / 4} D_{-2 v+1}(x) \text {. }
$$

The functions (3) and (4) were given by Mitra [2].

Taking the same function (1) for $f(x)$, we have, from (iii),

$$
g(x)=\int_{0}^{\infty} \frac{y^{\mu+1 / 2} e^{-x^{2} y^{2} / 2}(x y)^{\mu+1 / 2}}{\left(1+y^{2}\right)^{1+\mu / 2+\nu / 2}} d y=x^{\mu+1 / 2} \int_{0}^{\infty} \frac{y^{2 \mu+1} e^{-x^{2} y^{2} / 2}}{\left(1+y^{2}\right)^{1+\mu / 2+\nu / 2}} d y .
$$

Evaluating this integral by a formula given by Whittaker [8] we find that $g(x)$ is a constant multiple of 


$$
x^{(\mu+\nu-1) / 2} e^{x^{2} / 4} W_{-1 / 2-3 \mu ; 4-\nu / 4,(\nu-\mu) / 4}\left(x^{2} / 2\right),
$$

where $\mu>-1$.

Thus, we see that the function

$$
x^{\nu-2 m-1 / 2} e^{x^{2} / 4} W_{3 m-\nu-1 / 2, m}\left(x^{2} / 2\right),
$$

where $\nu-4 m>-1$, is $R_{\nu}$.

This function was given by Bailey [1].1

If we put the same function (1) for $f(x)$ in (i) and evaluate the corresponding integral by Hankel's formula [7], we arrive back at the $R_{\nu}$ function

$$
x^{\nu+1 / 2} e^{-x^{2} / 2} .
$$

If, in (iv), we take the same function for $f(x)$ we arrive at the function (6) again.

If we take the same function for $f(x)$ in $(v)$, we again arrive at the function (2).

\section{REFERENCES}

1. W. N. Bailey, Some classes of functions which are their own reciprocals in the Fourier-Bessel integral transform, Journal of the London Mathematical Society, vol. 5 (1930), pp. 258-265, (6.3).

2. - Self-reciprocal functions involving confluent hyper-geometric functions, ibid., vol. 13 (1938), pp. 111-112.

3. S. C. Dhar, On certain functions which are self-reciprocal in the Hankel transform, ibid., vol. 14 (1939), pp. 30-32.

4. W. T. Howell, A note on Laguerre polynomials, Philosophical Magazine, (7), vol. 23 (1937), pp. 807-811.

5. Brij Mohan (formerly B. M. Mehrotra), Some theorems on self-reciprocal functions, Proceedings of the London Mathematical Society, (2), vol. 34 (1932), pp. 231$240, \S 8$.

6. - On some self-reciprocal functions, Bulletin of the Calcutta Mathematical Society, vol. 25 (1933), pp. 167-172, §2.

7. G. N. Watson, Theory of Bessel Functions, Cambridge, 1922, $\S 13.3,(3)$ and 13.3, (4).

8. E. T. Whittaker and G. N. Watson, Modern Analysis, Cambridge, 4th edition, $1927, \$ 16.12$.

Benares Hindu University BENARES, INDIA

${ }^{1}$ Recently, Dhar [3] has given another proof of the fact that this function is $R_{\nu}$. The other $R_{\nu}$ function $x^{\nu+2 m-1 / 2} e^{x^{2} / 4} W_{-3 m-\nu-1 / 2, m}\left(x^{2} / 2\right)$ that he gives, is the same as (5) with " $-m$ " written for " $m$." 